\title{
LA ARTICULACIÓN DE LAS IDENTIDADES SOCIALES Y COLECTIVAS: UNA PERSPECTIVA SITUADA
}

\section{The articulation of social and collective identities: a situated perspective}

\author{
Juan Carlos Revilla*; Carlos de Castro Pericacho**; Francisco J. Tovar \\ Martínez*** \\ *Universidad Complutense de Madrid; ${ }^{* *}$ Universidad Autónoma de Madrid; ${ }^{* *}$ Universidad de \\ Valladolid \\ jcrevilla@cps.ucm.es; c.decastro@uam.es; fjtovar@soc.uva.es
}

\begin{abstract}
Resumen
El presente trabajo analiza las manifestaciones de las identidades colectivas que es posible encontrar en un determinado espacio social delimitado geográficamente, las ciudades alicantinas de Alcoy y Elda, partiendo de las identidades sociales más importantes. Se pretende contribuir a deslindar teóricamente ambos conceptos, los cuales no siempre han sido tratados adecuadamente. A través de entrevistas a expertos, en profundidad y grupos de

\section{Palabras clave}

Identidad colectiva Identidades laborales Identidades cívicas Campos sociales

\section{Keywords}

Collective identity Work-based identities Civic identities Social fields discusión, se ha realizado un análisis que identifica los más importantes discursos sobre el nosotros colectivo que encontramos en ambas ciudades, remitiéndonos a los campos sociales en los que se articulan las prácticas que les dan entidad y configuran esas identidades colectivas. El resultado muestra las dificultades para seguir articulando identidades colectivas a partir de las experiencias en la actividad productiva principal, al tiempo que emergen con fuerza unas identidades colectivas locales basadas en la participación en un espacio cívicopolítico que articula buena parte de la vida social.

\section{Abstract}

This paper analyzes the collective identities expressions that can be found in a geographically bounded social space, cities of Alcoy and Elda in Alicante, having as starting point the most important social identities. A contribution to the definition of bounds between both concepts, which have not been adequately treated, is intended. Through expert and in-depth interviews and focus groups, an analysis identifying most relevant discourses about the collective-we found in both cities has been developed, referring to the social fields that articulate the social practices which give significance and configure these collective identities. The results show the difficulties to keep articulating collective identities from experiences in the main productive activity, while local collective identities based on the participation in a civic-political space that articulates most of social life emerges.

Revilla, J.C., de Castro Pericacho, C., Tovar Martínez, F.J., 2015, "La articulación de las identidades sociales colectivas: una perspectiva situada", en Papeles del CEIC, vol. 2015/2, no 130, CEIC (Centro de Estudios sobre la Identidad Colectiva), Universidad del País Vasco, http://dx.doi.org/10.1387/pceic.14111
\end{abstract}




\section{INTRODUCCIÓN}

Una cuestión no bien resuelta en el análisis de las identidades son los términos en los que es posible referirse a identidades colectivas, como realidad diferente de las identidades individuales, pero, por supuesto, estrechamente conectadas. Una vía interesante de afrontar la cuestión sería analizar las posibilidades de articular identidades colectivas en un entorno social determinado, a partir de las identidades sociales más significativas. En la primera parte, trataremos de establecer un marco de comprensión de la identidad colectiva adecuado a nuestro objeto de estudio. En segundo lugar, buscaremos las posibilidades de configurar identidades colectivas en dos de los campos sociales más importantes de dos ciudades alicantinas (Elda y Alcoy), el campo de la producción industrial dominante y en crisis y el campo de la participación cívicopolítica. Por último, extraeremos algunas conclusiones más allá de los casos estudiados para contribuir al análisis de la relación compleja entre identidades individuales y colectivas.

Podríamos resumir en dos grandes perspectivas los estudios sobre identidad. En primer lugar, aquellos que parten del sujeto y tratan de describir y explicar los procesos involucrados en la dinámica de las identidades individuales. En segundo lugar, aquellos que centran su interés en determinadas identidades colectivas y buscan describirlas y/o explicar sus transformaciones.

La primera perspectiva goza de una salud extraordinaria, pues desde muy diferentes planteamientos se ha puesto de manifiesto su principal tesis, el carácter inherentemente social de la identidad individual (Íñiguez, 2001; Revilla, 1998). Los trabajos del Interaccionismo Simbólico (Stryker, 1980; Goffman, 1959; Mead, 1934) son un referente fundamental de la idea de que la interacción social es el lugar de producción de las identidades y de que ésta se construye desde los otros. El construccionismo social y el discursivismo (Edwards y Potter, 1992; Shotter y Gergen, 1989) añadieron el carácter narrativo de la identidad, lo que refuerza su carácter social, pues depende de discursos y de referentes sociales para configurarse. Por otro lado, los planteamientos estructuralistas, postestructuralistas y marxistas han enfatizado el carácter hegemónico de algunos discursos sociales (Gramsci, 1971) que reproducen un sistema de dominación, que se incorpora en las mentes y cuerpos de las personas a través de prácticas sociales que interpelan (Althusser, 1976) y constituyen a los sujetos que 
toman parte en ellas (Foucault, 1980). Los discursos del poder, pues, subjetivan (producen sujetos) al tiempo que sujetan (producen control social), al tiempo que diferencian, jerarquizan o excluyen (Foucault, 1975).

Por otra parte, algunos teóricos de la tardomodernidad entienden la identidad como un proyecto reflexivo ineludible en este orden postradicional (Giddens, 1991; Habermas, 1988), que se caracteriza por la complejidad y pluralidad de espacios de interacción, fragmentación de la experiencias sociales y multiplicación de las identidades posibles (Berger, 1967), lo que implica conceder un carácter activo a los procesos identitarios, implícito también en el concepto de construcción o en los planteamientos interaccionistas.

La segunda perspectiva ha producido también desarrollos muy importantes y presenta la enorme diversidad de las muy distintas identidades que ha analizado. Podriamos incluir los trabajos sobre identidades de género, muy centrados en la descripción y cuestionamiento del entramado simbólico que sostiene la discriminación de los grupos desfavorecidos (Haraway, 1989; Amorós, 1985), al igual que han cuestionado el sujeto del feminismo (ver Butler, 1990), ante la dificultad de hablar de una identidad común ante la pluralidad de experiencias subjetivas femeninas y adscripciones identitarias de las mujeres (de clase, etnia, sexual, etc.).

Igualmente, encontrariamos los trabajos sobre identidades nacionales, $\mathrm{u}$ otras identidades con base territorial, que han indagado sobre las bases de la construcción de la diferencia y sobre el reconocimiento político de la misma (Pérez-Agote 2008), eso sí, entendiendo las naciones como construcciones históricas y contingentes, no entidades naturales (Anderson, 1991; Hobsbawm, 1990; Gellner, 1983). La vinculación histórica entre identidades nacionales y étnicas ha motivado que ambas áreas sigan una trayectoria similar, con el triunfo de la tesis de las identidades étnicas como construcción histórica (Smith, 1986; Gellner, 1983). Las transformaciones sociales (migraciones) e institucionales (entidades supranacionales) están rompiendo las bases de las naciones-estado modernas y abriendo el camino a identidades nacionales más complejas o a plantear identidades territoriales múltiples o transnacionales (Benhabib, Shapiro y Petranovic, 2007). 
Son también clásicos los trabajos sobre el movimiento obrero, orientados hacia las injustas condiciones de vida y trabajo que han de traducirse en una movilización colectiva, lo que necesita de la articulación de una autopercepción como grupo, es decir, de la creación de una identidad obrera sólida (Dubar, 2000; Alonso, 1999; Sainsaulieu, 1977). La cuestión de la movilización ha sido importante para muchos investigadores que entienden la identidad colectiva como un elemento necesario para la acción colectiva (Melucci, 2001).

En cualquiera de estas perspectivas, la identidad colectiva no deja de presentar dificultades en su conceptualización. Berger y Luckmann (1967) ya previnieron del problema que suponía esencializar las identidades colectivas, pero tampoco resulta acertado entender la identidad colectiva como un aspecto de la identidad personal (Polletta y Jaspers, 2001), precisamente para evitar el riesgo de esencialización. Por otra parte, las modernas teorías de la identidad personal igualmente tienden a desesencializar o deconstruir al sujeto (Sampson, 1989), esto es, a criticar la noción de sujeto esencial, racional y autocontenido que caracteriza a la Psicología moderna, y a buena parte del pensamiento de sentido común.

Con una concepción de identidad colectiva como aspecto de la identidad personal se difumina el carácter colectivo de la misma y las supuestas identidades colectivas devienen no más que identidades compartidas. La consecuencia lógica no sería otra que la igualación con el concepto de identidad social (Turner, 1987; Tajfel, 1981), y plantear que los individuos en algunos casos actúan como sujetos individuales, en otros casos como miembros de un grupo y en otros como seres humanos, en función de la saliencia de la situación, que es justamente lo que plantea la teoría de la identidad social de Tajfel y Turner. Sin embargo, para Tajfel (1981), la identidad social se basa en la pertenencia del individuo a una categoría social determinada. Dado el carácter procesual de la perspectiva, no toma en consideración las elaboraciones simbólicas, presentes e históricas, que están detrás de los significados asociados con la pertenencia a diferentes colectivos sociales. Y solamente desde esa comprensión es posible entender la especificidad de las distintas colectividades $y$, con ello, comprender su actuación.

Tampoco parece adecuada la concepción marxista, que recuperan Martínez Lucio y Stewart (1997), que da por hecha la existencia de un trabajador colectivo (una identidad colectiva) por el hecho de que el 
proceso de trabajo es definido colectivamente. De esta forma, cualquier actuación de un trabajador implicaría una actuación colectiva. Coincidimos en la importancia de considerar la agencia de los trabajadores en cualquier entorno laboral y de que la organización colectiva del trabajo es una de las bases más importantes del carácter colectivo de esa agencia, pero no su asunción de que cualquier actuación de un trabajador implique una actuación colectiva, pues la agencia de los trabajadores puede ser meramente individual, en cuanto no compartida por otros o en cuanto a que puede perseguir intereses $u$ objetivos individuales y no colectivos. Por tanto, el carácter colectivo de la agencia es solamente potencial.

La pregunta sería entonces si el concepto de identidad colectiva aporta algo que sobrepase el carácter social de la identidad individual evitando el mencionado riesgo de esencialización o reificación, o si debemos dejar de distinguir entre identidad individual e identidad colectiva, pues todas las identidades serían colectivas. Entendemos que es necesario dejar un espacio específico para las identidades colectivas, como producto de la acción colectiva y de la autoconciencia como grupo, como realidad no esencial, sino emergente de un determinado momento de las relaciones sociales.

La identidad colectiva presenta dos aspectos fundamentales. En primer lugar, el hecho de saberse igual que otros, al tiempo que diferente de otros muchos que conforman grupos e identidades diferenciadas (Pujal, 2004; Iñiguez, 2001; Sainsaulieu, 1977). En segundo lugar, la definición compartida de lo que somos, un referente significativo común en el que nos reconocemos, al tiempo que por el que somos reconocidos (o representados, como señala Pérez-Agote, 2008). Esta definición compartida habrá de tener presencia en los discursos de los sujetos sociales, especialmente en los miembros del colectivo, pero también de algún modo en aquellos que lo tienen como identidad contrapuesta. Es ahí donde aparece un discurso del nosotros (y del ellos) que da lugar a relatos o narrativas de identidad colectiva, de modo no muy distinto a las de la identidad personal (ver Revilla, 1998). Los discursos del nosotros ponen de manifiesto el hecho de la identidad colectiva, esto es, el reconocimiento de la existencia de un colectivo identificable, por sus integrantes y desde fuera, así como las bases sobre las que se construye esa identidad. 
Si la identidad tiene carácter de construcción simbólica que se traducen en prácticas sociales, ha de guardar relación con otras construcciones simbólicas, como la cultura. Sin embargo, esto no debe conducir a la práctica igualación de cultura con identidad, pues esto minimiza las diferencias internas a todo grupo social y nos hace suponer que detrás de toda identidad colectiva existe necesariamente una cultura concreta y discernible de la de otras identidades colectivas. Esta tendencia es muy común en los estudios culturalistas sajones, por ejemplo, los que igualan cultura e identidad obreras. Una concepción más ajustada de la relación entre identidad y cultura pasaría por entender que cada cultura incorpora un sistema sancionado de distinciones, por utilizar la expresión de Saussure (1931) respecto del lenguaje, que enfatiza la importancia de los significados y los significantes lingüísticos, así como la relación de oposición entre los significados de los significantes del mismo ámbito, pues esta cuestión es igualmente fundamental para las dinámicas identitarias. Sin embargo, nos alejamos de la concepción de Saussure al entender que los significados no están fijados ni exentos de conflictos (ver Deetz, 1992). De esta forma, la cultura o la sociedad marcan el rango de identidades posibles y el significado que les resulta aplicable a las mismas. y tiene que ver igualmente con un sistema de posiciones sociales diferenciadas, propio del grupo, relacionado en buena medida con el sistema de distinciones. Estas posiciones sociales diferentes llevan asociadas diferentes habitus, en cuanto interiorización de normas sociales y de un universo cultural (Bourdieu, 1980).

En este sentido, podemos decir que los integrantes de una identidad colectiva comparten una cultura, pero no que los integrantes de una cultura compartan una sola identidad, sino que comparten una cosmovisión identitaria, es decir, una visión de quiénes y cómo son las personas que se adscriben o son situadas en diferentes identidades. A partir de ahí, dada la multiplicidad de identidades de cada sujeto, los individuos entran en dinámicas complejas de igualación y diferenciación, que en algunos casos se sitúan en el nivel interpersonal de la identidad social, pero en otros puede situarse en el nivel colectivo, no tanto por saliencia, como por posibilidad de actuación colectiva y por lógicas colectivas de acción.

Evidentemente, no es sencillo establecer una separación nítida, desde esta concepción, entre las manifestaciones de la identidad social y las de la identidad colectiva. Pero la distinción está clara en sus extremos. Es 
decir, no resulta difícil entender que cuando no existe apenas vinculación entre los diferentes sujetos consignados a una identidad social, o cuando las condiciones de existencia impiden o limitan su relación, no por ello deja de existir una configuración identitaria, un reconocimiento propio y externo en términos de sujetos individuales. Pensemos en los primeros emigrantes que acuden a un entorno extraño, en los trabajadores domésticos que viven aislados unos de otros y en condiciones laborales diferentes, incluso en el antropólogo que acude a una tribu extraña para su estudio. En el extremo de la identidad colectiva, se situarían aquellos colectivos que a partir de su identidad común han articulado unos criterios de actuación conjunta y han logrado una cierta cohesión que les lleva a comprometerse con ellos, siquiera temporalmente, lo cual suele tener que ver con unas condiciones de vida y/o intereses similares que les permiten además vincularse entre ellos. Aquí podríamos situar las identidades obreras bajo régimen fordista o en momentos de movilización colectiva, los movimientos sociales (feministas, ecologistas, etc.), las identidades territoriales (nacionales, regionales, locales) en momentos de celebración del nosotros o de confrontación y relación con los de fuera, etc. En estos ejemplos, se aprecia otra característica de las identidades colectivas, que no involucran en general a todos los integrantes de la identidad social que los reúne, pero quienes actúan en términos colectivos se sitúan metonímicamente como representantes del colectivo.

Por último, la dimensión diacrónica tiene una enorme importancia en la medida en que entendemos que las identidades están en continuo flujo. El sujeto individual modifica progresiva y al menos parcialmente los significantes con los que se identifica y/o es reconocido. Desde el punto de vista societal, los significantes identitarios relevantes pueden variar con el paso del tiempo, cambian las posibilidades de ser, pero también se modifica lo que entendemos por ser un tipo determinado tipo de persona (por ejemplo, no significa lo mismo ser profesor ahora que hace 50 años). Y dado el conjunto de identidades sociales relevantes, las posibilidades de articular una identidad colectiva variarán igualmente con el tiempo. Es decir, una identidad colectiva puede estar constituida en un momento determinado y desaparecer posteriormente si dejan de estar vigentes las condiciones simbólicas y estructurales que la hicieron posible. 


\section{LAS IDENTIDADES DE UN ESPACIO SOCIAL}

Entendemos necesaria una tercera perspectiva de acercamiento a la identidad que se haga cargo de la importante cuestión de la multiplicidad de identidades y de la interrelación compleja entre identidades sociales e identidades colectivas. Para apreciar estas dinámicas, sería interesante partir de un espacio social determinado en el que se interrelacionen diferentes actores sociales. Una posibilidad sería un espacio social delimitado geográficamente, sea en términos de país, región o ciudad. Evidentemente, cuanto menor el espacio, más sencillo conocer las dinámicas identitarias en su seno. y, si bien las identidades sociales posibles serán innumerables, será factible considerar las más relevantes $y$, desde luego, todas aquellas susceptibles de convertirse en identidades colectivas.

Entendemos que ésta es una perspectiva relativamente novedosa. No es novedoso ocuparse de las identidades con base territorial, pero normalmente esto se realiza dejando en segundo plano, si se llegan a considerar, las identidades de base no territorial. Otro antecedente cercano serían las monografías antropológicas de perspectiva holística, aunque normalmente no toman esta orientación identitaria, sino que han buscado la comprensión de los mecanismos de funcionamiento y configuración simbólica de la sociedad. Eso sí, sin desatender en muchos casos la existencia de diversidad interna, de estratificación social, lo que ya es un antecedente necesario para elaborar la multiplicidad de identidades.

Para acometer este análisis, necesitamos una concepción acerca de la pluralidad de la vida social que no deje de lado la cuestión de la estructuración social. Esto podría tratarse a través del concepto de campo (Bourdieu, 1980), entendido como un sector determinado de la actividad social, en el que los actores pertenecientes a un mismo grupo social desarrollan actuaciones diferentes en las diferentes esferas de acción social. Bourdieu (1991) define campo como una red de relaciones objetivas entre posiciones objetivamente definidas por su situación actual y potencial en la estructura de las distribuciones de las especies de capital (o de poder), cuya posición impone la obtención de beneficios específicos puestos en juego en el campo y, a la vez, por su relación objetiva con las otras posiciones. Por tanto, este concepto nos remite a la interacción social organizada en espacios sociales en los que entran en juego actores desde posiciones sociales diferentes. Según Bourdieu 
(1977), los diferentes grupos sociales estarían en lucha por el acceso a las mejores posiciones sociales para lo que ponen en juego no solo su capital económico, sino también sus capitales cultural, escolar y social. Además, la posición social ocupada por el grupo está detrás de diferencias en una serie de disposiciones perceptivas y prácticas (habitus) derivadas de la interiorización de las experiencias vividas como miembro del grupo de clase, por tanto, distintos universos de experiencias, ámbitos de prácticas y categorías de percepción y juicio.

Estos grupos que se relacionan en los campos sociales son sujetos de identidad social $y$, potencialmente, colectiva. Pero el hecho de que los actores que actúan en un campo social constituyan una identidad colectiva debe ser demostrado empíricamente. En línea con lo anterior, de la similitud cultural (habitus) o estructural (intereses) no tiene por qué seguirse la constitución de una identidad colectiva en los términos antes descritos. Por eso, en general es más sencillo hablar de identidades sociales.

Precisamente porque han sido muy diversos los campos analizados por Bourdieu y sus seguidores, no resulta sencillo establecer cuáles pueden ser los más significativos a la hora de producir identidades y quizá solo el conocimiento detallado del entorno social concreto puede ayudar a destacar algunos sobre otros. Quizá el problema radica en que Bourdieu no estableció una cierta taxonomía de los campos sociales, aunque contempló la existencia de subcampos sociales (Bourdieu, 2000).

Pero aparte de las identidades vinculadas a un campo social, creemos que es posible encontrar identidades no relacionadas con uno determinado, sino que serían significativas transversalmente, en todos, - la mayoría, de los campos sociales. En ese caso, situariamos a las identidades de género, de edad, las étnicas, incluso las religiosas. Así, las mujeres suelen ser interpeladas de forma diferente a los hombres en todos los campos sociales, del mismo modo que no significa lo mismo ser emigrante que autóctono para las posibilidades de sobrevivir en el mercado laboral.

Por tanto, a la hora de mirar las identidades en el marco de un espacio social determinado geográficamente, nos referiremos a dos tipos de identidades: las relacionadas con un campo social determinado, y que son irrelevantes fuera de las relaciones constituidas en el mismo, como las identidades laborales o las identidades políticas, y las transversales, 
que son relevantes en distintos campos sociales, como las ya mencionadas de género, edad o etnia, entre otras. Para ello, habremos de aportar algunos elementos característicos del campo, pero no realizaremos un análisis del campo à la Bourdieu.

Como marco para este análisis hemos seleccionado dos ciudades alicantinas $^{1}$. Alcoy y Elda forman parte de una serie de ciudades industriales especializadas de la provincia, como Villena, Elche, Ibi, etc. La industrialización de sus sectores productivos se produjo a finales del siglo XIX, siempre con retraso respecto de otros países europeos, en un patrón que dura hasta la actualidad, y sin abandonar totalmente el trabajo en el hogar, en una similar estrategia competitiva de reducción de costes. Ambas industrias experimentaron un crecimiento importante en los años 60, una nueva edad dorada, en Elda gracias a la exportación bajo la protección de compañías comercializadoras norteamericanas, en Alcoy debido sobre todo al incremento de la demanda interna. Las dos últimas décadas han visto el declive progresivo de la actividad en ambos sectores. Esta crisis prolongada se debe, en parte, a la liberalización y globalización de los mercados textil y de calzado, que ha hecho prácticamente imposible competir con los costes de producción de los países en vías de desarrollo. Este declive ha disparado una reflexión a nivel local acerca de la necesidad de reorientar estos sectores de producción hacia segmentos de producción de mayor calidad y valor añadido (Capó y Masía 2004).

Pero la actividad industrial ha significado para estas ciudades y sus ciudadanos mucho más que una actividad económica, también ha aportado un sentido de pertenencia a la ciudad y una identificación colectiva con esta producción y con el oficio. Desde el exterior, ambas ciudades son conocidas por esta actividad manufacturera. Desde el interior, los trabajadores pertenecen a una tradición industrial, y antes artesanal, lo que significa ser parte de una serie de valores y prácticas culturales y sociales.

Si bien ambas ciudades presentan diferencias significativas en cuanto a estructura social, mercado laboral, posibilidades de comunicación con el exterior, perspectivas de futuro del sector, etc., incluso lengua vernácula (ver Arnal et al., 2012), tienen también otro aspecto en común, una vida

\footnotetext{
${ }^{1}$ Este análisis forma parte del proyecto europeo SPHERE, financiado por la UE dentro del $7^{\circ}$ Programa Marco. 
ciudadana activa que se expresa a través de las celebraciones locales, especialmente las Fiestas de Moros y Cristianos. Es imposible en este trabajo hacer justicia a las diferentes tradiciones que, en ciencias sociales, se han ocupado del estudio de las fiestas populares (ver Ariño y García Pilán, 2006, para una revisión). Se ha atendido a su funcionalidad, digamos, antropológica (Mauss, 1925; Bataille, 1957), como mecanismo de control social y de subversión, controlada, del mismo (ver Delgado, 2004) y como una liberación transitoria y abolición provisional de privilegios que encuentra en la risa y en el exceso del carnaval su expresión más acabada (Bajtin, 1965). Se ha atendido a su carácter tradicional, que expresa la religiosidad popular de las comunidades rurales, sirviendo de elemento activador de identidades locales (Velasco, 2004), pero también a cómo las transformaciones sociales han incidido sobre la transformación del sentido de las fiestas, cambiando el sujeto festivo en muchos casos hacia colectivos urbanos (Ariño, 1992), lo que trastoca y complejiza los procesos de identidad implicados (García Pilán, 2011). En esta dirección, la clave estaría en cómo se leen las tradiciones históricas locales, ya sea como algo pasado que debe relegarse al olvido o como algo valioso que quizá se deba actualizar, o incluso convertir en parte del patrimonio histórico local, regional o nacional.

Todas estas cuestiones han estado presentes en las investigaciones llevadas a cabo específicamente sobre la fiesta de Moros y Cristianos. Se han rastreado sus orígenes históricos, $y$, en ese sentido, el significado profundo de la fiesta, vinculada al tiempo a fiestas religiosas populares como el Corpus o las fiestas patronales y a los enfrentamientos de soldadesca (Catalá, 2012; Sanchís y Sanchís, 2014). Se ha tratado de encontrar tipologías diferentes de fiesta (Catalá, 2012; Albert, 2003; Domene, 2006). Igualmente se ha investigado el proceso, no exento de conflictividad, de la patrimonialización de las fiestas, e incluso su apropiación política (Santamarina, 2008; Shefferman, 2014). Quizá se ha indagado menos acerca de la sociabilidad y las relaciones sociales que se generan alrededor de la preparación y celebración de las fiestas (Coloma, 1962; Mansanet, 2007), así como su papel en la generación de identidades locales (ver Tovar et al., 2011). Justamente estas últimas perspectivas son las más interesantes para este trabajo, pues indagaremos sobre las relaciones sociales en los campos sociales más relevantes de estas ciudades, al tiempo que indagaremos acerca de algunas de las identidades más relevantes en estos entornos sociales. 
Para ello, nos ocuparemos de dos de los campos sociales más relevantes, el campo de la producción y el campo de la participación cívico-política.

\section{Metodología}

El objetivo del proyecto consistía en analizar las transformaciones identitarias en ciudades o regiones que se han caracterizado por un predominio de un determinado tipo de actividad industrial que se encuentra en proceso de recesión o incluso de desaparición. Por eso, se ha buscado estudiar tanto las transformaciones de las identidades laborales como la posible emergencia de nuevas formas de identificación colectiva que pudieran adquirir relevancia de cara a posibles procesos de regeneración de la vida social y que eventualmente puedan servir de base para la puesta en marcha de procesos colectivos de revitalización social, cultural y, quizá también, económica en las ciudades bajo estudio.

Metodológicamente, el proyecto ha sido plural, si bien con predominio de las metodologías interpretativas, que han sido complementadas con análisis secundario de datos, como forma de acceder al contexto social general de los ámbitos de estudio. En concreto, se realizaron, entre los años 2009 y 2010, 20 entrevistas a expertos ${ }^{2}, 28$ entrevistas individuales en profundidad a ciudadanos de ambas ciudades (la mitad en cada una) y 3 grupos de discusión (2 en Elda y uno en Alcoy). Las personas entrevistadas fueron 14 mujeres y 14 hombres ( 7 en cada ciudad en ambos casos), con edades comprendidas entre los 18 y los 75 años ${ }^{3}$. Los grupos de discusión fueron dirigidos a jóvenes entre 20 y 35 años, con diferente vinculación con los sectores industriales tradicionales (calzado y textil), participando en total 10 hombres y 4 mujeres.

Además se recurrió a la observación participante de las fiestas locales más emblemáticas de ambas localidades (Moros y Cristianos), así como

\footnotetext{
${ }^{2}$ Los expertos fueron seleccionados entre académicos e investigadores regionales, informantes clave de los sectores textil y calzado (propietarios de fábricas, representantes sindicales), representantes de asociaciones culturales y religiosas locales, así como políticos y trabajadores públicos locales de ambas ciudades.

${ }^{3}$ Las personas entrevistadas fueron seleccionadas según su vinculación a las empresas del textil / calzado. Se realizaron entrevistas a trabajadores (13) o extrabajadores del sector (5 entrevistas), o trabajadores de otros sectores (10 entrevistas), con y sin vinculación familiar con el sector. Las entrevistas se segmentaron igualmente por edad: 7 entrevistas a jóvenes (de 20 a 35 años), 11 a adultos (entre 36 -55 años) y 10 mayores (56 años y más).
} 
numerosas visitas por los distintos barrios de las ciudades, polígonos y áreas industriales (antiguas y actuales). Con esta articulación de técnicas se pretendía acceder a diferentes perspectivas, orientaciones y experiencias respecto de la cuestión identitaria. El presente trabajo se nutre de todos estos materiales empíricos cualitativos (entrevistas, grupos de discusión, observación) que hemos producido en conjunción con expertos y habitantes de las dos ciudades.

\section{IDENTIDADES EN EL CAMPO DE LA PRODUCCIÓN INDUSTRIAL: ENTRE LA IDENTIDAD DE OFICIO Y LA IDENTIDAD OBRERA}

Las identidades en el campo de la producción industrial dominante se articulan en dos grupos-tipos principales de actores, empresarios y trabajadores, si bien tanto unos como otros pueden entrar y salir del campo (cambiar de sector de actividad). Desde el punto de vista identitario, en este campo nos encontramos con el empresario como identidad clave, mientras que, en cuanto a los trabajadores, son tres los tipos de identidad posibles: la identidad profesional u ocupacional, la identidad organizacional y la identidad obrera. Por la primera, nos referimos al reconocimiento derivado de saber desarrollar una actividad productiva, oficio o profesión, por tanto, una identidad social que distingue a unos trabajadores de otros, en un sector de actividad determinado, con unas determinadas características como profesional. No son escasos los trabajos que se han ocupado de este tipo de identidad, algunos ejemplos serían los trabajos sobre cuadros (Revilla y Tovar, 2009; Bouffartigue, 2001; Boltanski, 1982), sobre trabajadores del conocimiento (Westenholz, 2006), o sobre trabajadores temporales (Padavic, 2005), incluso Sennett (1998) no es ajeno a la consideración de ciertos grupos de trabajadores en cuanto profesionales. La identidad organizacional se refiere a la identificación de los trabajadores con la estructura que les acoge, en cuanto espacio normativo y simbólico. La preocupación por la identificación con la organización es un elemento emergente, pues desde las teorías manageriales se entiende que el compromiso con la organización es cada vez más importante como ventaja competitiva (Brown, 2001; Hogg y Perry, 2000). La identidad obrera se refiere al hecho de ser trabajador igual a otros y puede manifestarse a nivel de planta u organización, a nivel de sector o incluso a nivel regional o nacional. Existe un encendido debate en la Sociología del Trabajo acerca de cómo las nuevas formas de control organizacional 
estarian dificultando o transformando la formación de las identidades colectivas obreras de oposición a las imposiciones empresariales (Dubar, 2000; Martínez Lucio y Stewart, 1997; Edwards, Collinson y Della Rocca, 1995; Thompson y Ackroyd, 1995; Sainsaulieu, 1977).

Lo que vamos a analizar ahora es la interrelación entre esas identidades en las narrativas de los habitantes de nuestras poblaciones de estudio, en la medida en que reconstruyen un pasado, definen un presente e incluso imaginan un futuro.

Los discursos sobre los empresarios son muy distintos en ambos casos. En Alcoy, la imagen de los empresarios es marcadamente positiva, en la medida en que se les entiende como emprendedores comprometidos con el bienestar de la ciudad. Cierto es que, históricamente, los empresarios alcoyanos invirtieron en infraestructuras y servicios para la ciudad, como puentes, teatros, mutualidades, viviendas obreras, etc., lo cual les ha convertido en una especie de benefactores comprometidos con el futuro de la ciudad, no en vano pertenecen a la comunidad (Warner et al., 1963), lo cual no está exento del paternalismo que ha sido una característica histórica de la ciudad, y de la mano de una institucionalización de la negociación colectiva (explotadora, pero negociada). En estos momentos de práctica desaparición del sector, no se les responsabiliza de ello, sino más bien a la globalización y deslocalización empresarial. Sin embargo, ha existido también una percepción del empresariado como de un grupo coherente claramente diferenciado, una burguesía en el sentido sociológico del término.

Por el contrario, en Elda, la distancia entre los empresarios y los trabajadores es percibida como menor, y de hecho en muchos momentos el paso de trabajador a pequeño empresario no ha sido extraño. Pero el comportamiento de muchos empresarios es descrito como oportunista, que busca el lucro rápido y el enriquecimiento familiar y no la continuidad de la actividad empresarial. Además, las condiciones laborales son en general pésimas, dada la estrategia de reducción de costes que se ha empleado para mantener la competitividad, llegando a unas tasas de economía sumergida muy elevadas en el sector, lo que redunda en esa imagen de explotadores (piratas) de los empresarios. Bien es cierto que algunos empresarios se libran de esta imagen y son percibidos de forma positiva, lo que suele relacionarse con una apuesta por la producción de calidad y mejores condiciones laborales. 
Las identidades de los trabajadores han sufrido una suerte dispar en función igualmente de las características de sector y empresas. Dada la mayor mecanización de una producción textil poco intensiva en mano de obra, la identidad dominante en Alcoy ha sido una identidad clásica de obrero industrial, la identidad de oficio que describe Dubar (2000), vinculada con una estabilidad de las normas y la existencia de valores colectivos, que entra en crisis con el sector. Las estrategias empresariales para amortiguar el conflicto industrial potenciaron esta identidad al dirigirse, no tanto a mejorar las condiciones de trabajo en los talleres, sino al desarrollo de prácticas paternalistas a cambio de reducir las formas tradicionales de conflicto. Estas prácticas paternalistas se pensaban como una manera de "tejer" una identidad industrial y cultural local (Nash, 1989), subrayando la condición de "vecino de Alcoy" con la que todos los habitantes pueden identificarse.

La identidad trabajadora en el calzado eldense ha tenido un carácter más artesanal, pues, aún con la mecanización del proceso productivo, no se perdió del todo ese carácter artesanal del zapatero de silla. Solo en los momentos de apogeo del sector, en los años 60 y 70, cuando se manufacturaban millones de pares para las comercializadoras norteamericanas, se ha estado cerca en Elda de la configuración de una identidad clásica de obrero industrial. Pero la vinculación con el trabajo ha sido más individualista, quizá mejor decir familista, en la medida en que, si bien el hombre es el que solía trabajar en la fábrica, otros miembros de la familia, especialmente la mujer, colaboraban cosiendo (aparando) zapatos en el hogar familiar para la fábrica o para el taller propio. Por tanto, ha sido dominante una identidad de obrero-artesano, esto es, el orgullo por la experiencia y el conocimiento del arte de hacer zapatos. Este tipo de identidad puede ser la base de una identidad colectiva, como varios autores han mostrado (ver Dubar, 2000; Sainsaulieu, 1977). Pero en este caso la solidaridad requerida para una identidad común ha sido sustituida de algún modo por procesos de competencia entre trabajadores, especialmente cuando la transición de trabajador a propietario era más sencilla.

El análisis realizado hasta el momento muestra que en el caso de Alcoy las identidades colectivas han resultado más sencillas de conformar que en Elda, tanto las identidades de consenso, organizacionales, como las oposicionales. Sin embargo, no han sido extrañas las movilizaciones colectivas en esta última. La industrialización ayudó a crear las 
condiciones bajo las cuales apareció un vigoroso movimiento obrero que fue básicamente controlado por el anarcosindicalismo libertario en ambas ciudades (CNT; Beneito, Hernández y Molina, 2006; Valero et al., 1992) hasta la Guerra Civil. La represión franquista destruyó esta tradición y reforzó la identidad organizacional a través de las prácticas paternalistas que se reforzaron en el periodo de expansión productiva de los años 60 y 70 . Las excepcionales circunstancias de la transición política hicieron revivir las movilizaciones y reivindicaciones colectivas. En Alcoy, entre 1974 y 1976, se convocaron numerosas huelgas por estos aún ilegales sindicatos, que luchaban por mejorar las condiciones de trabajo (Moreno y Parra, 2007). En Elda se originó un espectacular movimiento asambleario en 1977 (Martínez, 1992), algo sorprendente en las condiciones bajo las cuales emergió (ver Tovar et al., 2011), pero muy arraigado en las transformaciones políticas del país y que revitalizó la tradición anarquista de otros tiempos. Sin embargo, la progresiva desindustrialización de nuestras ciudades ha conllevado un debilitamiento de la actividad sindical y de las movilizaciones obreras. De hecho, la última movilización colectiva ha sido protagonizada por obreros y empresarios que, esta vez juntos, exigían que sus actividades industriales recibieran la protección política del Estado, en línea con lo que planteaba Edwards (1979) acerca de cómo la clase obrera se vuelve hacia el gobierno para pedir protección y regulación.

El caso es que las experiencias colectivas obreras han fracasado en Elda, lo que dificultó la configuración de una identidad colectiva fuerte. Las condiciones que facilitaron la emergencia de una identidad colectiva, por ejemplo en los años 1960-70, fueron una cierta taylorización del sector, la percepción de posibilidades reales de mejorar las condiciones de trabajo, así como el extrañamiento entre trabajadores y propietarios. Es decir, los factores que explican en otros contextos la emergencia de movimientos de trabajadores pueden explicar aqui su discontinuidad y debilidad, en la medida en que no se dieron esos factores. La crisis recurrente de la industria fue en la dirección contraria: aumento del poder de las empresas, desregulación creciente, incremento de la economía sumergida, posibilidad de montar el propio "tallercico" o de abandonar hacia otros sectores de actividad (construcción hasta la crisis o turismo en la costa).

Todo esto ha provocado una desafección hacia la cultura industrial, especialmente en las generaciones más jóvenes. Ni siquiera los 
trabajadores recomiendan a sus hijos que permanezcan en el calzado. Por tanto, al contrario que en otros contextos, en los que los trabajadores lloran la pérdida de un pasado altamente valorado (ver Kirk, Jefferys y Wall, 2012), aquí están cada vez menos comprometidos con la tradición regional. En Alcoy, se produce precisamente esta nostalgia de un pasado duro, pero que permitió unas condiciones de vida dignas (reguladas), ante un presente incierto sin apenas industria textil, que impide cualquier identificación positiva y consolidada dentro de este campo social.

Pero lo más interesante de estos históricos momentos de vivencia de la identidad colectiva oposicional reside en que se convierten en un patrimonio cultural de los trabajadores, e incluso de la propia ciudad. Tanto sindicatos, como empresarios y ciudadanos recuerdan siempre esos acontecimientos históricos como reflejo de una tradición obrera importante que conforma la memoria colectiva de la ciudad. Así, la "Revolución del Petróleo" de 1873 en Alcoy o el movimiento asambleario de los años 70 en Elda están muy presentes en el imaginario colectivo de cada ciudad. Estos dos diferentes momentos comparten una característica importante: una elevada desconfianza hacia los sindicatos, de origen anarquista en el primer caso y más bien libertario en el segundo. Así, las industrias textil y del calzado continúan siendo un importante componente de la cultura de las dos ciudades, aunque corren el riesgo de que se conviertan en algo del pasado, que se mantiene más como reliquia cultural que como forma de vida.

Dentro de este campo productivo, dos son las identidades transversales que parecen más relevantes, en la medida en que generan diferencias significativas en el interior del campo: la identidad de género y las identidades nacional-regional-local.

En ambos sectores industriales, el trabajo de la mujer ha sido significativo y relevante. Sin embargo, la participación de la mujer no solía implicar su incorporación a los espacios públicos o al mercado de trabajo, pues la mayoría trabajaban en casa. Las mujeres tampoco participaban en actividades colectivas como sindicatos, algo más en los momentos importantes de movilización colectiva. Por tanto, siempre resultó una actividad subordinada al empleo del esposo, contribución secundaria a los ingresos de la casa. En esta situación es extremadamente difícil desarrollar un sentido de identidad personal basado en el trabajo industrial. Y cuando la tendencia general en España 
se ha dirigido hacia la incorporación de la mujer al mercado laboral (no sin dificultades, Fdez. Villanueva et al., 2003), las mujeres se han dirigido en nuestras ciudades hacia otros sectores laborales, por ejemplo, el comercio o los servicios, y no hacia los sectores industriales en crisis. Por ello, la identificación con la familia es predominante, incluso en las mujeres que tienen años de experiencia, especialmente como trabajadoras del calzado. Solo aquellas mujeres, más en Alcoy que en Elda, que han tenido un trabajo industrial equiparable a la norma masculina de trabajo tienden también a identificarse como trabajadoras industriales.

Tanto Elda como Alcoy han aumentado su población sustancialmente debido a la inmigración procedente de regiones agrícolas españolas: Castilla La Mancha, Andalucía y Extremadura, atraídas por la riqueza del sector industrial. Existe un discurso dominante en ambas ciudades de que la integración de estas poblaciones inmigrantes fue sencilla sin problemas importantes. Sin embargo, existe un discurso alternativo que enfatiza la segregación entre los de dentro y los de fuera. Aunque existían muchos trabajadores autóctonos, es cierto que éstos estaban en mejor disposición para regentar las empresas industriales, aunque en Elda existieron inmigrantes que llegaron a abrir talleres y eventualmente prosperaron convirtiéndose en empresarios reconocidos. La división más clara entre empresarios y trabajadores en Alcoy se refleja en una mayor diferenciación entre autóctonos y foráneos.

En cualquier caso, progresivamente esta diferenciación identitaria ha ido perdiendo sentido con el paso de dos generaciones, especialmente desde que ese flujo migratorio cesó y fue sustituido por la inmigración exterior. Pero dado que esta inmigración exterior se ha producido en tiempos ya de crisis industrial, los nuevos inmigrantes, en su gran mayoría, han permanecido fuera de este campo social al acceder a otros sectores de actividad (servicios y construcción).

\section{IDENTIDADES CÍVICAS Y POLÍTICAS}

Dada la crisis de las identidades basadas en el trabajo industrial, la existencia de otras identidades fuertes puede resultar crucial para mantener vínculos sociales sólidos que fijen a la población a este espacio social. En nuestro caso, que sería extensible a muchos otros lugares de nuestro país y otros, la pertenencia a un espacio político- 
territorial institucionalizado confiere precisamente unas posibilidades de identidad que son vividas intensamente por los ciudadanos. Es esa identidad derivada de la pertenencia a un territorio organizado políticamente en el municipio, pero también las identidades derivadas de las diferencias internas a ese espacio social, lo que nos remite a los diferentes grupos sociales relevantes en el mismo.

En ese sentido, podríamos denominar a este espacio el campo de la participación cívica y política. Cuando Bourdieu (2002) describe el campo político, establece una distinción clara entre los profesionales, los que forman parte del campo, y los profanos, que en puridad no forman parte de él, pero es a quienes los profesionales se dirigen para conseguir su favor. Justamente lo que nos interesa es el campo formado por esos profanos cuando participan cívica y/o políticamente en el espacio social, dejando fuera el campo propiamente político, que parece menos relevante para los procesos identitarios.

La identidad más relevante es la identidad común de miembros de la ciudad (ciudadanos), que iguala a todos los habitantes del espacio social, al tiempo que separa de los habitantes de otros territorios. Es, por tanto, uno de los tipos posibles de identidad con vinculación territorial (local, regional, nacional, supranacional), que están necesariamente relacionadas unas con otras. El hecho de centrarnos en el nivel local se debe a la fuerte presencia en nuestro material del discurso sobre la ciudad y sus habitantes. La producción discursiva sobre el nosotros local sale con mucha facilidad y se entiende como algo compartido. Vamos a analizar, pues, los componentes principales del discurso sobre el nosotros local (autorreconocimiento y reconocimiento externo), junto con los espacios y momentos sociales en que se traduce en prácticas sociales significativas.

El discurso de los alcoyanos sobre sí mismos enfatiza el orgullo de ser alcoyano, basado en su capacidad para hacer frente a unas condiciones geográficas adversas, lo que además se relaciona con el carácter emprendedor de la población que, sin apenas recursos naturales, ha sabido encontrar medios para la supervivencia colectiva. Un segundo elemento es el apego a las tradiciones propias de la ciudad, que se han preservado a lo largo de los siglos sin demasiadas variaciones y son parte de su patrimonio cultural. Incluso el patrimonio industrial se está intentando preservar en buena medida. Ambas circunstancias, además, 
distinguen a Alcoy de otras ciudades de la región, y de España, mucho menos apegadas a la tradición y el patrimonio hasta recientemente.

Los discursos autorreferidos de los eldenses contrastan vivamente. Sin dejar de sentir un cierto orgullo por su pertenencia a la ciudad, éste es en seguida relativizado, es decir, se manifiesta que cualquiera está orgulloso de su "pueblo" y que todo lugar presenta elementos positivos y negativos. Se manifiesta, igualmente, el carácter abierto y acogedor de la ciudad a personas de fuera, al tiempo que se menosprecia humorísticamente a los habitantes de las ciudades vecinas. Por otro lado, Elda no se caracteriza precisamente por la pervivencia de tradiciones de larga duración, se describen costumbres que aparecen, se ponen de moda y llegan a desaparecer. Como tampoco ha sido frecuente la conservación del patrimonio industrial de la ciudad, que ha sido engullido por su crecimiento.

Pero en lo que coinciden ambas ciudades, y muchas otras de la región, es en expresar esta identidad común de forma colectiva a través de las festividades locales. Son fiestas que se celebran todos los años y que estructuran una parte importante de los lazos sociales locales. Ambas otorgan la mayor importancia a las fiestas de Moros y Cristianos, pero socialmente desempeñan un papel similar al de las Fallas en muchas ciudades valencianas. Las fiestas de Moros y Cristianos alcoyanas, como corresponde a su identidad local, son seguramente las más antiguas de la provincia y se mantienen con la misma estructura generación tras generación. Las fiestas en Elda se recuperaron en los años 40 y progresivamente han ido incorporando novedades que las han ido diferenciado de las fiestas alcoyanas, en línea igualmente con su identidad local.

La importancia de la fiesta se traduce, por un lado, en que un gran número de habitantes de la ciudad participan en ella durante las celebraciones en sí mismas. Pero, también, la fiesta implica toda una organización social para su preparación que involucra igualmente a muchos habitantes. Durante la mayor parte del año, las comparsas eldenses y las filaes ${ }^{4}$ alcoyanas se reúnen regularmente para organizar el siguiente desfile o para realizar diversos actos relacionados. Las sedes de

\footnotetext{
${ }^{4}$ Las comparsas y las filaes son las agrupaciones o asociaciones que forman parte la fiesta de Moros y Cristianos, y que se encuadran en uno de los dos bandos y que representan a un grupo característico dentro de la representación con su identidad propia (ver Catalá, 2012).
} 
estos grupos se reparten por el casco antiguo, siendo un lugar de reunión social para sus miembros, amigos y familiares, no solo durante las fiestas. Por ello, la participación en la red social festera supone una oportunidad para convertir la pertenencia a la localidad en prácticas cotidianas estructuradas que refuerzan precisamente el vínculo colectivo y son un momento magnífico de manifestación de la identidad colectiva. En estos tiempos de crisis, estas celebraciones, por los vínculos sociales que implican, pueden tener mayor fuerza identitaria que las industrias tradicionales, especialmente entre los jóvenes o en los trabajadores expulsados del sector. El sentimiento de pertenencia a la ciudad $y$, en consecuencia, el deseo de permanecer cuando las perspectivas no son buenas, se crea y se mantiene mediante la participación en las fiestas. Por ello, las fiestas pueden estar actuando como un anclaje identitario o un factor de cohesión e identificación colectiva.

Estas prácticas sociales están abiertas a todos los habitantes de la ciudad, que pueden decidir si participar o no, o con más o menos intensidad, pero el resultado conjunto es el de la vivencia de la identidad colectiva. De hecho, la implicación de una persona o familia en la fiesta puede ser variable o diferente. Por ejemplo, los jóvenes tienden a vivir más el ocio nocturno, mientras que las familias con hijos pequeños tienden a implicarles en los desfiles infantiles o en otras actividades diurnas. Incluso se habla de personas que se fueron de la ciudad, pero que vuelven para vivir la fiesta (y rememorar su identidad local).

Por otro lado, la participación en la fiesta refleja de algún modo la estructura social de la ciudad en cada caso, además de una diferente relación con la actividad industrial dominante, constituyendo de hecho dos modelos diferentes de organización de la fiesta (Albert, 2003). En Alcoy, tradicionalmente los miembros de la burguesía empresarial textil ocupaban los puestos de privilegio en las filaes y en los desfiles, sufragando buena parte de los gastos comunes, en una especie de potlatch (Mauss, 1925) urbano. Pero, al mismo tiempo, las filaes se organizan de forma totalmente democrática e igualitaria, con la participación, literalmente codo con codo, de directivos, empleados y obreros, lo que históricamente ha servido para encauzar los conflictos industriales en el interior del grupo. En Elda, la participación está menos vinculada con los actores del calzado, al menos no hay percepción de una implicación especial. La organización de la fiesta permite compartir 
los gastos comunes que se derivan de la misma, de forma que el principal gasto de los participantes tiene que ver con su atuendo personal y su participación en diversos actos festivos, etc. Por eso, se acerca a lo que Veblen (1902) denominaba consumo ostentoso, unos gastos que muestran el poder económico personal y familiar.

Sin embargo, las tradiciones alcoyanas están empezando a modernizarse: por ejemplo, ahora cualquier persona puede ser capitán de la filà, aunque no se pertenezca a una familia de propietarios industriales, si puede sufragar los gastos, aunque sea a crédito. Y los gastos comunes y de los cortejos están empezando a ser sufragados por todos los miembros de la filà, dadas las dificultades de las empresas del sector.

En cuanto a las identidades transversales, el carácter tradicional de las fiestas alcoyanas las convierte en un espacio social más cerrado para ciertos grupos poblacionales. Las fiestas, como actividad pública, han sido un espacio fundamentalmente masculino, en el que la participación de la mujer estaba muy restringida (ver también Gisbert, 2012). Y aun hoy las mujeres no pueden desfilar conjuntamente con los hombres ni pueden ser miembros regulares de la filà. Por el contrario, en Elda las mujeres participan en plena igualdad con los hombres en las comparsas, tienen los mismos derechos y pueden desfilar conjuntamente, aunque en la práctica pueda apreciarse cierta segregación en los desfiles. Pero por costumbre social, no por imposibilidad normativa. Y, acorde con los tiempos, la mujer adquiere un protagonismo especial en la vistosidad de unos vestidos que refuerzan su feminidad y sensualidad. Por otro lado, la presencia de la mujer es importante en ambos casos en los actos religiosos que se celebran alrededor de las fiestas, lo que es un signo más de su papel tradicional.

¿Y qué sucede con la participación de los foráneos en las fiestas? La naturaleza de la organización social de las mismas conlleva que quienes no están integrados en el tejido social difícilmente puedan participar con normalidad. En Alcoy, la forma de ingresar en la fiesta era, sobre todo, a través del trabajo industrial. En Elda, la mayor flexibilidad en los procesos de integración social implica también una mayor dificultad, al depender de relaciones sociales y de la entrada en las redes sociales de la ciudad. Además, en cualquiera de los dos casos, resulta difícil vivir una fiesta que implica tanto gasto e implicación a quienes les resultan extraños esos rituales. De hecho, muchos inmigrantes aprovechaban las 
fiestas para pasar unos días en sus localidades de origen, lo que también dificultaba la vivencia de sus hijos de la fiesta. Por eso, encontramos ese discurso en los hijos de inmigrantes, la desimplicación de sus padres de la fiesta y una cierta frustración por no poder participar, como sus compañeros, o su sentimiento de no pertenecer del todo a la ciudad. En sentido contrario, la participación en la fiesta se convierte en un símbolo de la integración social del inmigrante, de su deseo de formar parte de la comunidad local.

\section{CONCLUSIONES}

El ejercicio de análisis que hemos realizado no hace más que apuntar a algunas, quizá las más relevantes, de las posibles identidades colectivas en nuestras dos ciudades. El análisis podría ir más allá a atender otros campos sociales, que articularian a la población en torno a otros ejes, dando lugar a otras identidades sociales significativas que, eventualmente, podrían servir a la formación de identidades colectivas. Un ejemplo podría ser el campo religioso. Pero incluso el campo de la participación cívico-política queda incompleto, pues cabría analizar las identidades políticas propiamente dichas o la organización territorial de las ciudades en barrios más o menos organizados a través de sus asociaciones de vecinos.

Pero el caso es que el análisis realizado hasta aquí ha permitido poner en evidencia nuestra pretensión inicial, que no era otra que analizar las dinámicas de las identidades colectivas más relevantes en dos ciudades alicantinas. Las identidades colectivas son una producción sujeta a las eventualidades de los espacios sociales, de forma que determinadas circunstancias de los mismos pueden hacer posible o, por el contrario, dificultar, su aparición. De este modo, hemos visto cómo la posibilidad de configurar una identidad colectiva obrera entra en crisis con la crisis del sector tras un pasado en el que la movilización obrera y/o la regulación fordista del trabajo (incluso en condiciones de trabajo exigentes) la pusieron en el primer plano social de la ciudad. Hemos visto cómo esa identidad colectiva obrera, especialmente en Alcoy, articulaba buena parte la estructura social de la ciudad, siendo relevante en otros campos sociales. Eso sí, la desaparición de una identidad colectiva fuerte no significa que las identidades sociales derivadas de la actividad laboral dejen de ser importantes en cuanto identidad social ni que no sea posible la aparición de manifestaciones débiles de la 
identidad colectiva pretérita. De hecho, la pervivencia de lo que fue la identidad obrera puede servir como elemento de anclaje para nuevas movilizaciones obreras, al menos en Elda, donde la actividad industrial se mantiene hasta cierto punto. Eso sí, hemos visto también que la práctica desaparición del sector textil en Alcoy lleva aparejada inevitablemente la casi desaparición del campo social donde era posible sostener identidades vinculadas con la producción y el trabajo industrial. En cuanto a Elda, el sostenimiento, precario y semisumergido, del sector del calzado conduce a la desafección con cualquier identidad que emerja de él. Es difícil identificarse con identidades sociales no percibidas como valiosas, no por el trabajo en sí, sino por las condiciones en que se realiza. De ahí la renuencia de los eldenses a trabajar en el sector del calzado si tienen una mejor opción. En los dos casos, los nuevos sectores de actividad proveen de distintas identidades sociales vinculadas con el trabajo, seguramente más frágiles, pero difícilmente de una revitalización de las identidades colectivas laborales, dada la fragmentación de la actividad y la experiencia laborales que es característica, en general, del mercado de trabajo en el régimen postfordista.

Por el contrario, la consolidación de las instituciones democráticas, el debilitamiento de las identidades de clase y la mejora general del nivel de vida de las poblaciones españolas se puede relacionar con el desarrollo de las identidades colectivas locales. De hecho, en la organización social alrededor de las fiestas, aunque goza de gran tradición, especialmente en Alcoy, entendemos que el grado de implicación de diferentes capas de la población ha aumentado y con ello se ha convertido metafóricamente a las fiestas de Moros y Cristianos en el alma de la ciudad, en una demostración de lo que somos como colectividad y de cómo queremos ser vistos desde fuera. Por tanto, se convierte claramente en una identidad colectiva valiosa con la que identificarse.

Las identidades colectivas que se forman según esta concepción y este análisis no constituyen en absoluto entidades esenciales inamovibles, ni se entiende que todos los sujetos que se identifican con una categoría inevitablemente formarán parte de ese colectivo. Resulta más adecuado, también más complejo, entender la identidad colectiva como manifestada en prácticas sociales que implican un grado de reflexividad conjunta que una vez constituida adquiere un reconocimiento externo 
que puede resultar atractivo y servir de polo atractor para integrantes de la categoría social en la que se basa la identidad colectiva. Pero, al tiempo, los sujetos que forman parte del colectivo pueden entrar y salir de esas prácticas sociales o tener una implicación parcial, sin que eso suponga necesariamente un menoscabo para la colectividad. En ese sentido, consideramos que una vez constituida, la identidad colectiva se convierte en un recurso al que se pueden acercar o del que se pueden beneficiar diferentes sujetos que forman parte de la identidad social de base. De este modo, con esta concepción se pueden entender las dinámicas de los movimientos sociales, por ejemplo, el feminista, que en sus mejores momentos atrajo a mujeres que podian tener más dificultades para integrar el movimiento y así constituido beneficiarse todas de las reivindicaciones colectivas.

\section{BibLIOGRAFÍA}

Albert, J.-P., 2003, “Moros y cristianos en el País Valenciano: la ilusión de la fiesta", en M.A. Llorca y J.A. González Alcantud (Eds.), Moros y Cristianos, Presses Universitaries du Mirail, Toulouse, pp. 89-102.

Alonso, L.E., 1999, Trabajo y ciudadanía. Estudios sobre la crisis de la sociedad salarial, Ed. Trotta, Madrid.

Althusser, L., 1976, "Idéologie et appareils idéologiques d'État”, en L. Althusser, Positions (1964-1975), Les Éditions sociales, París, pp. 67125.

Amorós, C., 1985, Hacia una crítica de la razón patriarcal, Anthropos, Barcelona.

Anderson, B., 1991, Imagined Communities: reflections on the origin and spread of nationalism, Verso, London.

Ariño, A., 1992, La ciudad ritual. La fiesta de las Fallas, Anthropos, Barcelona.

Ariño, A., García Pilán, P., 2006, "Apuntes para el estudio social de la fiesta en España", en Anduli, 6, pp. 13-28.

Arnal, M., de Castro, C., Lahera-Sánchez, A., Revilla, J.C., Tovar, F.J., 2012, "Two Spanish cities at the crossroads: changing identities in Elda and Alcoy", en J. Kirk, S. Jeffreys y S. Contrepois (Eds.), Changing work and community identities in European regions: perspectives on past and present, Palgrave, London, pp. 91-123.

Bajtin, M., 1965, La cultura popular en la Edad Media y en el Renacimiento. El contexto de François Rabelais, Alianza, Madrid. 
Bataille, G., 1957, El erotismo, Tusquets, Barcelona.

Beneito, Á., Hernández, R., Molina, R., 2006, “El siglo XIX: época de cambios y revoluciones", en J. Santonja y J. Segura (Eds.), Historia de Alcoy, Ayuntamiento de Alcoy, Editorial Marfil y Centre Alcoià d'Estudis Històrics i Arqueòlogics, Alcoy, pp. 265-293.

Benhabib, S., Shapiro, I., Petranovic, D., 2007, Identities, Affiliations, and Allegiances, Cambridge U. Press, Cambridge.

Berger, P., 1967, El dosel sagrado. Elementos para una sociología de la religión, Amorrortu, Buenos Aires.

Berger, P., Luckmann, T., 1967, La construcción social de la realidad, Amorrortu, Madrid.

Boltanski, L., 1982, Les cadres. La formation d'un groupe social,Minuit, Paris.

Bouffartigue, P. (Dir.), 2001, Cadres: la grande rupture, La Découverte, Paris.

Bourdieu, P., 1977, La reproducción, Laia, Barcelona.

Bourdieu, P., 1980, La distinción, Madrid, Taurus.

Bourdieu, P., 1991, "Le champ littéraire", en Actes de la Recherche en Sciences Sociales, 89, pp. 4-46.

Bourdieu, P., 2000, Les structures sociales de l'économie, Seuil, Paris.

Bourdieu, P., 2002, Propos sur le champ politique, Presses Universitaires de Lyon, Lyon.

Brown, A.D., 2001, "Organization studies and identity: towards a research agenda", en Human Relations, 54(1), pp. 113-121.

Butler, J., 1990, Gender Trouble. Feminism and the subversion of identity, Routledge, London.

Capó, J., Masía, E., 2004, "Evolución del sector textil en España: el caso textil-hogar", en Economía Industrial, 355-356, pp. 283-304.

Catalá, D., 2012, "La fiesta de moros y cristianos: herencia cultural compartida entre España y América Latina", en A. Colomer (Ed.), América Latina, Globalidad e Integración, Ediciones del Orto, Madrid, pp. 407-426.

Coloma, R., 1962, Libro de la fiesta de moros y cristianos de Alcoy, Instituto Alcoyano de Cultura 'Andrés Sempere', Alcoy.

Deetz, S., 1992, "Disciplinary power in the Modern corporation", en M. Alvesson y H. Willmott (Eds.), Critical management studies, Sage, London, pp. 21-45. 
Delgado, M., 2004, "Tiempo e identidad. La representación festiva de la comunidad y sus ritmos", en Zainak, 26, pp. 77-98.

Domene, J.F., 2006, “Elementos y tipología de Fiestas de Moros y Cristianos", en J.F. Domene, M.A. González y V. Vázquez (Eds.), Las fiestas de Moros y Cristianos en el Vinalopó, Centre d'Estudis Locals del Vinalopó, Petrer.

Dubar, C., 2000, La crisis de las identidades: la interpretación de una mutación, Bellaterra, Barcelona.

Edwards, R.C., 1979, Contested terrain: The transformation of the workplace in the Twentieth Century, Basic Books, New York.

Edwards, P., Collinson, D., Della Rocca, G., 1995, “Workplace Resistance in Western Europe: A Preliminary Overview and a Research Agenda", en European Journal of Industrial Relations, 1(3), pp. 283-316.

Edwards, D., Potter, J., 1992, Discursive Psychology, Sage, London.

Fernández, C., Domínguez, R., Revilla, J.C., Anagnostou, A., Sancho, M., 2003, La igualdad de oportunidades: los discursos de las mujeres sobre avances, obstáculos y resistencias, Icaria, Barcelona.

Foucault, M., 1975, Surveiller et punir, Gallimard, Paris.

Foucault, M., 1980, Power/Knowledge, Harvester Wheatsheaf, Brighton.

García Pilán, P., 2011, “Rituales, descentramientos territoriales y niveles de identidad: La Semana Santa Marinera de Valencia", en Revista de Dialectología y Tradiciones Populares, LXVI, 2, pp. 355-374.

Gellner, E., 1983, Nations and nationalism, Ithaca, Cornell U. Press, United States.

Giddens, A., 1991, Modernity and self-identity: self and society in the late modern age, Polity Press, London.

Gisbert, V., 2012, “Feminidades y Masculinidades en la Fiesta de Moros y Cristianos de Alcoi", en Prisma Social. Revista de Ciencias Sociales, 7, pp. 92-119.

Goffman, E., 1959, La presentación de la persona en la vida cotidiana, Amorrortu, Madrid.

Gramsci, A., 1971, El materialismo histórico y la filosofía de Benedetto Croce, Nueva Visión, Buenos Aires.

Habermas, J., 1988, Pensamiento postmetafísico, Taurus, Madrid.

Haraway, D.J., 1989, Primate visions: Gender, race and nature in the world of modern science, Routledge, Nueva York. 
Hobsbawm, E.J., 1990, Nations and Nationalism Since 1780, Cambridge University Press, Cambridge.

Hogg, M.A., Perry, D.J., 2000, "Social Identity and self-categorization processes in organizational contexts", en Academy of Management Review, 25(1), pp. 121-140.

Iñiguez, L., 2001, "Identidad: de lo personal a lo social. Un recorrido conceptual", en E. Crespo y C. Soldevilla (Eds.), La constitución social de la subjetividad, Libros de la Catarata, Madrid, pp. 209-226.

Kirk, J., Jefferys, S., Wall, C., 2012, "Representing Identity and Work in Transition: The case of South Yorkshire Coal-Mining Communities in the UK", en J. Kirk, S. Jeffreys y S. Contrepois (Eds.), Changing work and community identities in European regions: perspectives on past and present, Palgrave, London, pp. 184-216.

Mansanet, J.L., 2007, Las Fiestas de Moros y Cristianos: una selección de cuarenta años de artículos del autor sobre la temática festera, Diputación de Alicante, Alicante.

Martínez, F., 1992, “Desarrollo económico y cambio social: La industria del calzado en Elda 1950-1980", en J. R. Valero et al. (Eds.), Elda, 18321980: Industria del calzado y transformación social, Instituto de Cultura Juan Gil-Albert, Alicante.

Martínez Lucio, M., Stewart, P., 1997, "The Paradox of Contemporary Labour Process Theory: The Rediscovery of Labour and the Disappearance of Collectivism", en Capital and Class, 62(2), pp. 49-78.

Mauss, M., 1925, "Ensayo sobre el don: forma y función del intercambio en las sociedad arcaicas", en Sociología y Antropología, Tecnos, Madrid.

Moreno, F., Parra, M., 2007, La resistencia antifranquista y las Comisiones Obreras en las comarcas del sur del País Valenciá, Editorial Germanía, Valencia.

Mead, G.H., 1934, Espiritu, persona y sociedad, Paidos, México.

Melucci, A., 2001, Challenging codes, Cambridge U. Press, Cambridge.

Nash, J.,1989, From tank town to high tech, SUNY, Albany.

Padavic, I., 2005, "Laboring under uncertainty: Identity renegotiation among contingent workers", en Symbolic Interaction, 28(1), pp. 111134.

Pérez Agote, A., 2008, Las raíces sociales del nacionalismo vasco, CIS, Madrid.

Pujal, M., 2004, "La identidad (self)", en T. Ibáñez et al. (Eds.), Introducción a la Psicología Social, UOC, Barcelona, pp. 93-138. 
Polletta, F., Jaspers, J.M., 2001, "Collective identity and social movements", en Annual Review of Sociology, 27, pp. 283-305.

Revilla, J.C., 1998, La identidad personal de los jóvenes: pluralidad y autenticidad, Entinema, Madrid.

Revilla, J.C., Tovar, F.J., 2009, "La (re)producción narrativa de la identidad laboral y sus condiciones de posibilidad", en E. Crespo, C. Prieto y A. Serrano (Eds.), Trabajo, subjetividad y ciudadania: paradojas del empleo en una sociedad en transformación, Ed. Complutense, Madrid, pp. 113-138.

Sainsaulieu, R., 1977, L'identité au travail, Presses de la Fondation Nationale des Sciences Politiques, $3^{a}$ Edición, Paris.

Sampson, E.E., 1989, "The deconstruction of the self", en J. Shotter y K.J. Gergen (Eds.), Texts of identity, Sage, London, pp. 1-19.

Sanchis, R., Sanchis, V., 2014, "Más de un siglo de embajadas humorísticas entre moros nous y estudiants en Banyeres de Mariola", en Actas del I Congreso de Internacional de Embajadas. Disponible en: http://issuu.com/societatdefesters/docs/congreso_embajadas_part e1. Última visita: 05/06/2015.

Santamarina, B., 2008, "Moros y Cristianos: De la Batalla Festiva a la Discursiva", en Gazeta de Antropología, 24 (1), http://www.ugr.es/ pwlac/G24_16Beatriz_Santamarina_Campos.ht $\underline{\mathrm{ml}}$.

Saussure, F. de, 1931, Curso de Lingüística General, Alianza, Madrid.

Sennett, R., 1998, La corrosión del carácter. Las consecuencias del trabajo en el nuevo capitalismo, Anagrama, Barcelona.

Shefferman, D.A., 2014, "Rhetorical Conflicts: Civilizational Discourse and the Contested Patrimonies of Spain's Festivals of Moors and Christians", en Religions, 5, pp. 126-156.

Shotter, J., Gergen, K.J. (Eds.), 1989, Texts of identity, Sage, London.

Smith, A.D., 1986, The Ethnic Origins of Nations, Blackwell, Oxford.

Stryker, S., 1980, Symbolic interactionism: a social structural versión, Benjamin-Cummings, Menlo Park.

Tajfel, H., 1981, Grupos humanos y categorías sociales, Herder, Barcelona.

Thompson, P., Ackroyd, S., 1995, "All Quiet on the Workplace Front? A Critique of Recent Trends in British Industrial Sociology", en Sociology, 29(4), pp. 615-33. 
Tovar, F.J. et al., 2011, "A tale of two cities: working class identity, industrial relations and community in declining textile and shoe industries in Spain", en International Journal of Heritage Studies, 17, 4, pp. 331-343.

Turner, J.C., 1987, Redescubrir el grupo social, Morata, Madrid.

Valero, J.R. et al., 1992, Elda, 1832-1980: Industria del calzado y transformación social, Instituto de Cultura Juan Gil-Albert, Alicante.

Veblen, T., 1902, Teoría de la clase ociosa, FCE, México.

Velasco, H.M., 2004, "Fiestas del pasado, fiestas para el futuro", en P. Martínez-Burgos García y A. Rodríguez González (Coords.), La fiesta en el mundo hispánico, Cuenca, Universidad de Castilla-La Mancha, pp. 43-68.

Warner, W.L. et al., 1963, Yankee City (abridged edition), Yale University Press, New Haven.

Westenholz, A., 2006, "Identity work and meaning arena. Beyond actor/structure and micro/macro distinctions in an empirical analysis of IT workers", en American Behavioral Scientist, 49, 7, pp. 1015-1029. 